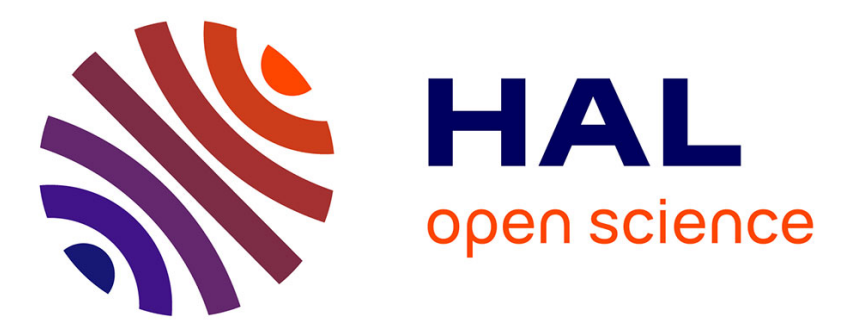

\title{
Forwarding design with prescribed local behavior
}

Sofiane Benachour, Vincent Andrieu, Laurent Praly, Hassan Hammouri

\section{To cite this version:}

Sofiane Benachour, Vincent Andrieu, Laurent Praly, Hassan Hammouri. Forwarding design with prescribed local behavior. IEEE Transactions on Automatic Control, 2013, 58 (12), pp.3011 - 3023. 10.1109/TAC.2013.2277632 . hal-00793554

\section{HAL Id: hal-00793554 https://hal.science/hal-00793554}

Submitted on 26 Feb 2013

HAL is a multi-disciplinary open access archive for the deposit and dissemination of scientific research documents, whether they are published or not. The documents may come from teaching and research institutions in France or abroad, or from public or private research centers.
L'archive ouverte pluridisciplinaire HAL, est destinée au dépôt et à la diffusion de documents scientifiques de niveau recherche, publiés ou non, émanant des établissements d'enseignement et de recherche français ou étrangers, des laboratoires publics ou privés. 


\title{
Forwarding design with prescribed local
}

\section{behavior}

\author{
M.Sofiane Benachour, Vincent Andrieu, Laurent Praly and Hassan Hammouri
}

\begin{abstract}
Among the non-linear control techniques, some Lyapunov design methods (Forwarding / Backstepping) take advantage of the structure of the system (Feedforward-form / Feedback-form) to formulate a continuous control law which stabilizes globally and asymptotically the equilibrium. In addition to stabilization, we focus on the local behaviour of the closed loop system, providing conditions under which we can predetermine the behaviour around the origin for Feedforward systems.
\end{abstract}

\section{Index Terms}

Stabilization, Lyapunov design, Forwarding, First order approximation, Feedforward form.

\section{INTRODUCTION}

The synthesis of a stabilizing control law for systems described by nonlinear differential equations has been the subject of great interest by the nonlinear control community during the last three decades. Depending on the structure of the model, some techniques are now available to synthesize control laws ensuring global and asymptotic stabilization of the equilibrium point.

For instance, we can refer to the popular backstepping approach (see [1] and the reference therein), or the forwarding approach (see [2], [3], [4], [5]) and some others based on energy considerations (see [6] for a survey of the available approaches).

L. Praly Laurent.Praly@ensmp. fr is with the Centre d'Automatique et Systèmes, Mines ParisTech, 35 Rue Saint Honoré, 77305 Fontainebleau, France.

M.S. Benachour, V. Andrieu and H. Hammouri are with Université de Lyon, F-69622, Lyon, France; Université Lyon 1, Villeurbanne; CNRS, UMR 5007, LAGEP (Laboratoire d'Automatique et de Génie des Procédés). 43 bd du 11 novembre, 69100 Villeurbanne, France benachouralagep.univ-lyon1.fr https://sites.google.com/site/vincentandrieu/, hammouri@lagep.univ-lyon1.fr 
Although the global asymptotic stability of the equilibrium point can be achieved in some specific cases, it remains difficult to address at the same time, performance issues of a nonlinear system in a closed loop. However, when the first order approximation of the non-linear model is considered, some performance aspects can be addressed by using linear optimal control techniques (using LQ controller for instance).

Hence, it is interesting to raise the question of synthesizing a nonlinear control law which guarantees the global asymptotic stability of the origin while ensuring a prescribed local linear behavior. This type of question has been already discussed in the literature when backstepping design is used to synthesize a nonlinear continuous control law (see [7]).

In the present paper, we consider the same problem in the case of a system whose structure allows forwarding design techniques (see [3], [4])).

The paper is organized as follows. In Section II-A, the problem under consideration is described. Section II-B is devoted to the statement of the main theorem and to its discussion in the case of systems that are obtained after adding some dynamics composed of a stable part and integrations. Section IV gives an illustration of the results on a class of systems composed of a quadratic nonlinear part with a linear subsystem. Finally, Section V gives the conclusion.

\section{PROBLEM DESCRIPTION AND GENERAL RESUlT}

\section{A. Problem description}

To present the problem under consideration, we introduce a general controlled nonlinear system described by the following ordinary differential equation:

$$
\dot{\chi}=\Phi(\chi, u)
$$

with the state $\chi$ in $\mathbb{R}^{N}$ and $\Phi: \mathbb{R}^{N} \times \mathbb{R}^{m} \rightarrow \mathbb{R}^{N}$ is a $C^{1}$ function such that $\Phi(0,0)=0$ and $u$ is a control input in $\mathbb{R}^{m}$. For this system, we can introduce the two matrices describing its first order approximation at the origin which is assumed to be stabilizable:

$$
\mathscr{A}_{0}:=\frac{\partial \Phi}{\partial \chi}(0,0), \mathscr{G}:=\frac{\partial \Phi}{\partial u}(0,0)
$$

For system (1), the problem we intend to solve can be described as follows:

Stabilization with prescribed local behavior: Let the linear state feedback law $u=K_{o} \chi$ 
stabilizing the first order approximation of system (1) be given. We are looking for a stabilizing control law $\alpha_{o}: \mathbb{R}^{N} \rightarrow \mathbb{R}^{m}$, differentiable at 0 such that:

1) the origin of the system:

$$
\dot{\chi}=\Phi\left(\chi, \alpha_{o}(\chi)\right)
$$

is globally and asymptotically stable.

2) The first order approximation of the control law $\alpha_{o}$ satisfies:

$$
\frac{\partial \alpha_{o}}{\partial \chi}(0)=K_{o}
$$

A general answer to this problem has been given in [8], requiring the system to be input affine. However, the set of local linear controllers $K_{o}$ are those which satisfy a specific linear matrix inequality. Adding some structural constraints on the system (1) this problem has been addressed in [7] where the system is in strict feedback form.

In our study, we consider the case in which by decomposing the state as $\chi=[y, x]$ the system (1) can be rewritten in the following Feedforward form.

$$
\left\{\begin{array}{l}
\dot{y}=A y+h(x), \\
\dot{x}=f(x)+g(x) u,
\end{array}\right.
$$

with $y$ in $\mathbb{R}^{n_{y}}, x$ in $\mathbb{R}^{n_{x}}$ and with $f: \mathbb{R}^{n_{x}} \rightarrow \mathbb{R}^{n_{x}}, g: \mathbb{R}^{n_{x}} \rightarrow \mathbb{R}^{n_{x} \times m}$ and $h: \mathbb{R}^{n_{x}} \rightarrow \mathbb{R}^{n_{y}}$ are $C^{p}$ functions, $p \geq 2$ such that $h(0)=0$ and $f(0)=0$ and $u$ is the control input in $\mathbb{R}^{m}$.

The stabilization problem for this class of system has been deeply studied in the last two decades employing forwarding techniques (see for instance [2], [3], [4], [5], [9], [10]). Compared to our preliminary result in [11], the novelty comes from the fact that $y$ is not a scalar.

The first order approximation of system (2) is denoted:

$$
\left\{\begin{array}{l}
\dot{y}=A y+H x, \\
\dot{x}=F x+G u,
\end{array}\right.
$$

with the matrices $H, F$ and $G$ given as

$$
F=\frac{\partial f}{\partial x}(0), H=\frac{\partial h}{\partial x}(0), G=g(0) .
$$

In the following, we make three structural Assumptions on the nonlinear system (2). The first one establishes that the first order approximation is stabilizable.

Assumption 1: The system (3) is stabilizable. 
The second assumption we make is also a local property and concerns more specifically the vector field $g(x)=\left(g_{1}(x), \ldots, g_{m}(x)\right)$.

Assumption 2: The distribution Vect $\left\{g_{1}(x), \ldots, g_{m}(x)\right\}$ is involutive and of constant dimension $m$ in a neighborhood of the origin.

In the case where there is only one input (i.e. $m=1$ ), this assumption is always satisfied provided $g(0) \neq 0$.

In the spirit of [3], we make the following assumption on the matrix $A$ in the $y$ subsystem.

Assumption 3: There exists a positive definite matrix $P$ in $\mathbb{R}^{n_{y} \times n_{y}}$ such that the following equality holds

$$
P A+A^{\prime} P=0
$$

This Assumption implies that the matrix $A$ has all its eigenvalues with zeros real part and we recover the case in which $y$ is scalar as already studied in our preliminary conference paper [11].

Also, we assume that the stabilization problem with any prescribed local behavior can be solved for the $x$ subsystem in system (2). More precisely, given $p$ in $\mathbb{N}$ we make the following assumption on the functions $f$ and $g$ :

Assumption 4: For all matrix $K_{x}$ in $\mathbb{R}^{m \times n_{x}}$ such that the matrix $F+G K_{x}$ is Hurwitz, there exists a function $\alpha_{x}: \mathbb{R}^{n_{x}} \rightarrow \mathbb{R}^{m}$ of class $C^{p}$ such that the following two properties are satisfied:

1) the origin of the system:

$$
\dot{x}=f(x)+g(x) \alpha_{x}(x) .
$$

is globally and asymptotically stable;

2) the first order approximation of this function satisfies:

$$
\frac{\partial \alpha_{x}}{\partial x}(0)=K_{x}
$$

\section{B. Main result}

We are now ready to state the main result which gives sufficient conditions guaranteeing that the stabilization with prescribed local behavior can be solved for system (2).

Theorem 1 (Adding integration with prescribed local behavior): Assume the System (2) satisfies Assumptions 1, 2, 3 and 4 for a given $p$ in $\mathbb{N}$. Given a linear controller $K_{o}=\left(K_{o, y}, K_{o, x}\right)$ 
in $\left(\mathbb{R}^{m \times n_{y}} \times \mathbb{R}^{m \times n_{x}}\right)$ such that the matrix:

$$
\mathscr{A}=\left[\begin{array}{cc}
A & H \\
G K_{o, y} & F+G K_{o, x}
\end{array}\right],
$$

is Hurwitz. Assume moreover that there exists a positive definite matrix $\mathscr{P}$ defined as

$$
\mathscr{P}=\left[\begin{array}{cc}
P & Q \\
Q^{\prime} & R
\end{array}\right]
$$

which satisfies the weak Lyapunov inequality

$$
\left[\mathscr{P} \mathscr{A}+\mathscr{A}^{\prime} \mathscr{P}\right] \leq-c_{L}\left[\begin{array}{cc}
Q G G^{\prime} Q^{\prime} & 0 \\
0 & S
\end{array}\right],
$$

and where $P$ is a positive definite matrix in $\mathbb{R}^{n_{y} \times n_{y}}$ which satisfies (5) and where $S$ is a $n_{x} \times n_{x}$ positive definite matrix and $c_{L}$ is a positive real number and $Q G$ is left invertible. Then there exists a $C^{p-1}$ function $\alpha_{o}: \mathbb{R}^{n_{x}+n_{y}} \rightarrow \mathbb{R}^{m}$ such that the following properties are satisfied :

1) the function $\alpha_{o}$ satisfies:

$$
\frac{\partial \alpha_{o}}{\partial y}(0,0)=K_{o, y}, \frac{\partial \alpha_{o}}{\partial x}(0,0)=K_{o, x}
$$

2) the origin of the system

$$
\dot{y}=A y+h(x), \dot{x}=f(x)+g(x) \alpha_{o}(x, y),
$$

is globally stable ${ }^{1}$. Moreover, if any forward bounded solution to the system

$$
\dot{y}=A y, \quad y^{\prime} Q G=0,
$$

defined on $[0+\infty[$, converges to the origin then the origin is globally asymptotically stable.

\section{Discussion on Theorem 1}

1) About Assumption 4 : Assumption 4 is stronger than a stabilizability property since it is assumed that all local behaviors can be recovered for the closed loop system. However, employing the result obtained in [7], yields that Assumption 4 is satisfied in the case in which the $x$ subsystem is in strict feedback form and when the functions $f$ and $g$ are sufficiently smooth. Note also that it is trivially satisfied when this system is a linear controllable system as studied in Section IV.

\footnotetext{
${ }^{1}$ An equilibrium point is said globally stable if it is stable and if all solutions are bounded (see [12, Page 40] for further details).
} 
2) About the weak linear Lyapunov inequality:

The right hand side of inequality (10) may not be a full rank matrix. Indeed, this one is of rank $n_{x}+m$. In order to apply Theorem 1, we need to find $\mathscr{P}$ solution to the weak Lyapunov inequality (10) and (5). Note that in the case there is one input (i.e. $m=1$ ), this construction can be reformulated in terms of an equivalent linear matrix inequality. Indeed, we can show the following proposition.

Proposition 1: Let $K_{o}=\left[K_{o, y}, K_{o, x}\right]$ in $\mathbb{R}^{n}$ be given such that $K_{o, y} \neq 0$. Let $P$ be a positive definite matrix in $\mathbb{R}^{n_{y} \times n_{y}}$ which satisfies (5). The matrix $\mathscr{P}$ defined in (9) satisfies (10) with $Q G \neq 0$ if and only if it satisfies the following linear matrix inequality

$$
\mathscr{P}>0,\left[\mathscr{P} \mathscr{A}+\mathscr{A}^{\prime} \mathscr{P}\right] \leq-c_{L}\left[\begin{array}{cc}
K_{o, y}^{\prime} K_{o, y} & 0 \\
0 & S
\end{array}\right], S>0 .
$$

Proof: Assume (14) is satisfied. Since $K_{o, y} \neq 0$ this implies that $Q G \neq 0$. Moreover with (5), $\mathscr{P} \mathscr{A}+\mathscr{A}^{\prime} \mathscr{P} \leq 0$ implies that $Q G K_{o, y}+K_{o, y}^{\prime} G^{\prime} Q^{\prime}$ is a negative semidefinite matrix. For this property to hold, this implies that there exists a positive real number ${ }^{2} \lambda$ such that $Q G=-\lambda K_{o, y}^{\prime}$. Consequently (10) is satisfied. The proof that (10) implies (14) follows the same lines.

In the case in which $n_{y}=1$ (this implies that $A=0$ ) this assumption can always be satisfied (indeed, this is the usual Lyapunov inequality). However, this is not the case when $n_{y}>1$. For instance, if we consider the case of a system whose first order approximation is a linear system of the form

$$
\left\{\begin{array}{l}
\dot{y}_{1}=-y_{2} \\
\dot{y}_{2}=y_{1}+x \\
\dot{x}=u
\end{array}\right.
$$

It is shown in Appendix A that for all stabilizing linear controllers in the form $u=k_{2} y_{2}+k_{x} x$ where $\left(k_{2}, k_{x}\right)$ are real numbers (i.e. $K_{o}=\left[K_{o, y}, K_{o, x}\right]$ with $\left.K_{o, y}=\left[0, k_{2}\right]\right)$, it is not possible to find $\mathscr{P}$ such that the weak Lyapunov inequality (10) is satisfied with $Q G \neq 0$. Hence, our approach can't be applied for this stabilizing local control law.

\footnotetext{
${ }^{2}$ Given two real vectors $v_{1}$ and $v_{2}$ of the same dimension such that the matrix $v_{1} v_{2}^{\prime}+v_{2} v_{1}^{\prime}$ is negative semidefinite. Note that we have, $\left(v_{1} v_{2}^{\prime}+v_{2} v_{1}^{\prime}\right)\left(\left|v_{2}\right| v_{1}+\left|v_{1}\right| v_{2}\right)=\left(\left|v_{2}\right| v_{1}+\left|v_{1}\right| v_{2}\right)\left(v_{1}^{\prime} v_{2}+\left|v_{1}\right|\left|v_{2}\right|\right)$. Consequently, $\left(v_{1}^{\prime} v_{2}+\left|v_{1}\right|\left|v_{2}\right|\right)$ is an eigenvalue which is strictly positive unless $v_{1}^{\prime} v_{2}=-\left|v_{1}\right|\left|v_{2}\right|$. Hence the result.
} 
3) About the result: As mentioned in the previous comment, when $n_{y}=1$, the weak linear Lyapunov inequality is satisfied for all stabilizing linear controller. Consequently when $n_{y}=1$, the conclusion of Theorem 1 implies that Assumption 4 is valid for the entire system with stabilizer in $C^{p-1}$. Hence, with an iterative procedure, higher order systems can be considered. Indeed, let system (1) be with $\chi=(y, z, x)$, with $z=\left(z_{1}, \ldots, z_{n_{z}}\right)$ in the form:

$$
\left\{\begin{array}{l}
\dot{y}=A y+h(x, z) \\
\dot{z}_{1}=h_{1}\left(z_{2}, \ldots, z_{n_{z}}, x\right) \\
\vdots \\
\dot{z}_{n_{z}}=h_{n_{z}}(x) \\
\dot{x}=f(x)+g(x) u
\end{array}\right.
$$

with $x$ in $\mathbb{R}^{n_{x}}, z_{i}$ in $\mathbb{R}$ and $y$ in $\mathbb{R}^{n_{y}}, f: \mathbb{R}^{n_{x}} \rightarrow \mathbb{R}^{n_{x}}, g: \mathbb{R}^{n_{x}} \rightarrow \mathbb{R}^{m}$ and $h_{i}: \mathbb{R}^{n_{z}-i+n_{x}} \rightarrow \mathbb{R}$ are $C^{i+2}$ functions, such that $h_{i}(0, \ldots, 0)=0$ and $f(0)=0$ and $u$ is the control input in $\mathbb{R}$. Based on the result obtained from Theorem 1, we can show the following result:

Theorem 2 (Case of higher order systems): Assume the $x$ subsystem of (16) satisfies Assumptions 2 and 4 with $p=n_{z}+2$. Assume moreover the first order approximation of this system is stabilizable. For all vector $\left(K_{o, y}, K_{o, z}, K_{o, x}\right)$ in $\left(\mathbb{R}^{m \times n_{y}} \times \mathbb{R}^{m \times n_{z}} \times \mathbb{R}^{m \times n_{x}}\right)$ which stabilizes globally and asymptotically the first order approximation of system (16) and such that there exists a matrix $\mathscr{P}$ in the form (9) with $Q$ in $\mathbb{R}^{n_{y} \times\left(n_{x}+n_{z}\right)}$ which satisfies (10) with $Q G$ left invertible then there exists a $C^{1}$ function $\alpha_{o}: \mathbb{R}^{n_{y}+n_{z}+n_{x}} \rightarrow \mathbb{R}^{m}$ such that the following properties are satisfied :

1) the function $\alpha_{o}$ satisfies:

$$
\frac{\partial \alpha_{o}}{\partial y}(0,0,0)=K_{o, y}, \frac{\partial \alpha_{o}}{\partial z}(0,0,0)=K_{o, z}, \frac{\partial \alpha_{o}}{\partial x}(0,0,0)=K_{o, x} ;
$$

2) the origin of the system (16) in closed loop with $u=\alpha_{o}(y, z, x)$ is globally stable and if moreover the origin is the only solution to the system (13) then the origin is globally asymptotically stable.

Proof: First, employing Theorem 1 it is shown that the $\left(z_{n_{z}}, x\right)$-subsystem in system (16) satisfies Assumption 4 with $p=n_{z}+1$. Recursively, we apply again Theorem 1 and we obtain the result.

In the paper [8], the stabilization with prescribed local behavior has been addressed and studied on an inverted pendulum model. In some specific coordinates, this inverted pendulum model can 
be put in forwarding form and a forwarding control law has been introduced in [3]. It is noticed in [8] that, statistically, for all local behavior obtained from a $L Q$ approach, the stabilization with prescribed local behavior could be solved. Consequently, Theorem 1 establishes a theoretical justification on the fact that the approach of [8] applies on the forwarding model of the inverted pendulum.

\section{PRoOF OF THEOREM 1}

The proof of this result is divided into four parts. In the first part, we focus on the linear approximation of the system and we show that the quadratic Lyapunov function associated with the local stabilizer (i.e. $\mathscr{P}$ ) can be rewritten in the form of a Lyapunov matrix that would have been obtained by following the forwarding design method of [3]. In the second part of the proof, we construct a candidate Lyapunov function $V$ for the nonlinear model such that its quadratic approximation is the matrix $\mathscr{P}$. In the third part, from this candidate Lyapunov function we construct a control law which makes non positive the time derivative of the candidate Lyapunov function. By interpolating this control law with the local controller, we finally get our solution to the stabilization with prescribed local behavior. Finally, in the fourth part, we construct a Lyapunov function associated to this control law and show that LaSalle invariance principle may be applied to get asymptotic convergence of the closed loop trajectories toward the origin.

\section{A. Part 1: Forwarding local Lyapunov function}

In this part of the proof, we show that the weak Lyapunov matrix $\mathscr{P}$ associated with the matrix $\mathscr{A}$ can be rewritten in the form of a Lyapunov matrix that would have been obtained following the Forwarding design method of [3] or [5].

Indeed, note that the Lyapunov function associated to the matrix $\mathscr{P}$ can be decomposed as follows.

$$
\left[\begin{array}{ll}
y^{\prime} & x^{\prime}
\end{array}\right] \mathscr{P}\left[\begin{array}{l}
y \\
x
\end{array}\right]=x^{\prime} R_{x} x+(y-M x)^{\prime} P(y-M x),
$$

where $R_{x}$ and $M$ are respectively a matrix in $\mathbb{R}^{n_{x} \times n_{x}}$ and a vector in $\mathbb{R}^{n_{x}}$ defined as:

$$
R_{x}=R-Q^{\prime} P^{-1} Q, M=-P^{-1} Q .
$$

If we compare the decomposition in equation (17) and the structure of the Lyapunov function obtained by the forwarding technique of [3] (see [5, equation (3)]), we see that the matrix $\mathscr{P}$ 
would be a Lyapunov matrix obtained by a forwarding design technique provided there exists $K_{x}$ a vector in $\mathbb{R}^{n_{x}}$ such that the following two requirements are satisfied:

1) There exists a matrix $K_{x}$ in $\mathbb{R}^{m \times n_{x}}$ which may differ from $K_{o, x}$ and such that the following algebraic equation is satisfied (see [3, equation (132)]):

$$
M\left(F+G K_{x}\right)=A M+H .
$$

2) $u=K_{x} x$ is a control law for the $x$-subsystem associated to the Lyapunov matrix $R_{x}$. In other words, $F+G K_{x}$ is a Hurwitz matrix and the following inequalities are satisfied :

$$
R_{x}>0, R_{x}\left(F+G K_{x}\right)+\left(F+G K_{x}\right)^{\prime} R_{x}<0
$$

In this part of the proof we show that a vector $K_{x}$ satisfying (19) and (20) does exist. Indeed, we can decompose $\mathscr{A}=\mathscr{A}_{0}+\mathscr{G} K_{o}$ with $^{3}$

$$
\mathscr{A}_{0}=\left[\begin{array}{cc}
A & H \\
0_{n, 1} & F
\end{array}\right], \mathscr{G}=\left[\begin{array}{l}
0 \\
G
\end{array}\right] \text {. }
$$

With these notation, we have

$$
\mathscr{P} \mathscr{G}=\left[\begin{array}{c}
Q G \\
R G
\end{array}\right]
$$

and, employing the fact that $P A+A^{\prime} P=0$ we get

$$
\mathscr{P} \mathscr{A}_{0}+\mathscr{A}_{0}^{\prime} \mathscr{P}=\left[\begin{array}{cc}
0 & P H+Q F+A^{\prime} Q \\
Q^{\prime} A+H^{\prime} P+F^{\prime} Q^{\prime} & Q^{\prime} H+R F+H^{\prime} Q+F^{\prime} R
\end{array}\right] .
$$

Since we have assumed that $Q G$ is left invertible we can introduce the two matrices respectively in $\mathbb{R}^{n_{y} \times n_{x}}$ and $\mathbb{R}^{n_{y} \times n_{y}}$

$$
w_{1}=-Q G\left(G^{\prime} Q^{\prime} Q G\right)^{-1} G^{\prime} R, w_{2}=Q G\left(G^{\prime} Q^{\prime} Q G\right)^{-1} G^{\prime} Q^{\prime}-I
$$

Note that we have

$$
w_{1}^{\prime} Q G=-R G, w_{2}^{\prime} Q G=0
$$

Hence, we have for all $n_{x} \times n_{y}$ real matrices $\beta$,

$$
v^{\prime} \mathscr{P} \mathscr{G}=0, v=\left[\begin{array}{c}
w_{1}+w_{2} \beta \\
I_{n_{x}}
\end{array}\right] .
$$

${ }^{3}$ the symbol $0_{a, b}$ stands for a $a \times b$ zero matrix. 
Note moreover that we have

Hence, it implies with (10)

$$
v^{\prime}\left[\begin{array}{cc}
Q G G^{\prime} Q^{\prime} & 0 \\
0 & S
\end{array}\right] v=S+R G G^{\prime} R>0 .
$$

$$
v^{\prime}\left[\mathscr{P} \mathscr{A}+\mathscr{A}^{\prime} \mathscr{P}\right] v<0
$$

This yields recalling that $\mathscr{A}=\mathscr{A}_{0}+\mathscr{G} K_{o}$ and with (22)

$$
v^{\prime}\left[\mathscr{P} \mathscr{A}_{0}+\mathscr{A}_{0}^{\prime} \mathscr{P}\right] v<0
$$

More precisely, the following inequality is satisfied for all $\beta$

$$
\begin{aligned}
& v^{\prime}\left[\mathscr{P} \mathscr{A}_{0}+\mathscr{A}_{0}^{\prime} \mathscr{P}\right] v=\left[\beta^{\prime} w_{2}^{\prime}\left(P H+Q F+A^{\prime} Q\right)+\left(H^{\prime} P+F^{\prime} Q^{\prime}+Q A\right) w_{2} \beta\right] \\
&+\left[w_{1}^{\prime}\left(P H+Q F+A^{\prime} Q\right)+\left(H^{\prime} P+F^{\prime} Q^{\prime}+Q A\right) w_{1}+Q^{\prime} H+R F+H^{\prime} Q+F^{\prime} R\right]<0
\end{aligned}
$$

The matrix inequality (24) being true for all matrices $\beta$, it is for instance true for $\beta=\alpha w_{2}^{\prime}(P H+$ $\left.Q F+A^{\prime} Q\right)$ where $\alpha$ is a positive real number. Hence we get

$$
\begin{aligned}
\alpha^{2}\left[w_{2}^{\prime}(P H+\right. & \left.\left.Q F+A^{\prime} Q\right)\left(H^{\prime} P+F^{\prime} Q^{\prime}+Q A\right) w_{2}\right] \\
& +\left[w_{1}^{\prime}\left(P H+Q F+A^{\prime} Q\right)+\left(H^{\prime} P+F^{\prime} Q^{\prime}+Q A\right) w_{1}+Q^{\prime} H+R F+H^{\prime} Q+F^{\prime} R\right]<0 .
\end{aligned}
$$

Letting $\alpha$ goes to infinity implies that we have

$$
w_{2}^{\prime}\left(P H+Q F+A^{\prime} Q\right)=0 .
$$

This gives,

$$
Q G\left(G^{\prime} Q^{\prime} Q G\right)^{-1} G^{\prime} Q^{\prime}\left(P H+Q F+A^{\prime} Q\right)-P H-Q F-A^{\prime} Q=0
$$

Consequently it yields

$$
-Q\left[F+G\left[-\left(G^{\prime} Q^{\prime} Q G\right)^{-1} G^{\prime} Q^{\prime}\left(P H+Q F+A^{\prime} Q\right)\right]\right]=A^{\prime} Q+P H .
$$

Left multiplying the previous equality by $P^{-1}$ and employing the fact that with (5) we have $P^{-1} A^{\prime}=-A P^{-1}$ it yields

$$
-P^{-1} Q\left[F+G\left[-\left(G^{\prime} Q^{\prime} Q G\right)^{-1} G^{\prime} Q^{\prime}\left(P H+Q F+A^{\prime} Q\right)\right]\right]=A\left[-P^{-1} Q\right]+H .
$$

Hence, we recover equation (19) with $M=-P^{-1} Q$ and

$$
K_{x}=-\left(G^{\prime} Q^{\prime} Q G\right)^{-1} G^{\prime} Q^{\prime}\left(P H+Q F+A^{\prime} Q\right) .
$$


It remains to check if $K_{x}$ satisfies (20). Note that (24) and (25) imply that

$$
w_{1}^{\prime}\left(P H+Q F+A^{\prime} Q\right)+\left(H^{\prime} P+F^{\prime} Q^{\prime}+Q A\right) w_{1}+Q^{\prime} H+R F+H^{\prime} Q+F^{\prime} R<0 .
$$

Replacing $w_{1}$ by its definition given in (21) in the previous inequality yields,

$$
R\left(F+G K_{x}\right)+\left(F+G K_{x}\right)^{\prime} R+Q^{\prime} H+H^{\prime} Q<0 .
$$

On another hand, with (27), we have

$$
Q^{\prime} H=-Q^{\prime} P^{-1} Q\left[F+G K_{x}\right]-Q^{\prime} A P^{-1} Q .
$$

Hence (29) becomes employing again $P^{-1} A^{\prime}=-A P^{-1}$,

$$
S_{x}:=R_{x}\left(F+G K_{x}\right)+\left(F+G K_{x}\right)^{\prime} R_{x}<0 .
$$

Therefore, for the time being, we showed this surprising property.

Lemma 1: Let $\mathscr{P}$ be a matrix in the form (9) solution to the weak Lyapunov inequality (10) and such that its upper left block is a Lyapunov matrix associated to $A$ (i.e. (5) is satisfied). Then this matrix can be decomposed in a forwarding-like manner. In other words, $M$ and $R_{x}$ defined in (18) satisfy (19) and (20).

From this crucial property, we will be able to get a candidate Lyapunov function for the nonlinear system associated to the local controller.

\section{B. Part 2: Construction of the global CLF}

In this part of the proof we construct a global (weak) control Lyapunov function denoted $V_{g}$ for the nonlinear system (2) and such that its Hessian satisfies ${ }^{4} \mathscr{H}\left(V_{g}\right)(0)=2 \mathscr{P}$. The construction of the candidate Lyapunov function is based on a modified forwarding technique inspired from [3] and employs Assumption 4. First, with Assumption 4, and the local stabilizer $K_{x}$ given in (28), there exists a $C^{p}$ function $\alpha_{x}: \mathbb{R}^{n} \rightarrow \mathbb{R}^{m}$ such that the origin of the system (6) is globally and asymptotically stable and the local property (7) is satisfied.

Now, we can apply the following Lemma whose proof is given in Appendix B.

Lemma 2: There exists a $C^{\infty}$ Lyapunov function $V_{x}: \mathbb{R}^{n} \rightarrow \mathbb{R}_{+}$, proper and positive definite, such that:

\footnotetext{
${ }^{4}$ The symbol $\mathscr{H}$ denotes the operator which gives the Hessian of a given $C^{2}$ function in $\mathbb{R}^{n}$.
} 
- $V_{x}$ is a Lyapunov function associated to the closed loop system (6). In other words, we have:

$$
-W_{x}(x):=\frac{\partial V_{x}}{\partial x}(x)\left[f(x)+g(x) \alpha_{x}(x)\right]<0, \forall x \neq 0 ;
$$

- $V_{x}$ is locally quadratic and its local approximation is $R_{x}$ defined in (18). We have:

$$
\mathscr{H}\left(V_{x}\right)(0)=2 R_{x} .
$$

For the non linear system (2), following the forwarding design described in [13] and [3], we can introduce the function $\mathscr{M}: \mathbb{R}^{n_{x}} \rightarrow \mathbb{R}^{n_{y}}$ defined as:

$$
\mathscr{M}(x)=\int_{+\infty}^{0} \exp (-A s) h\left(X_{1}(x, s)\right) d s,
$$

where $X_{1}(x, s)$ is the solution initiated from $x$ and evaluated at time $s$ of the system:

$$
\dot{x}=f(x)+g(x) \alpha_{x}(x) .
$$

The following Lemma can be obtained from [13, Lemma 6.88]. See also [4, Secction 5.2] et [3].

Lemma 3 ([13]): The function $\mathscr{M}$ defined in (33) is zero at the origin, $C^{p}$ and satisfies the following partial differential equation:

$$
\frac{\partial \mathscr{M}}{\partial x}(x)\left[f(x)+g(x) \alpha_{x}(x)\right]=A \mathscr{M}(x)+h(x), \forall x \in \mathbb{R}^{n} .
$$

With $V_{x}$ obtained from Lemma 2, the function $\mathscr{M}$ given in (33) we consider the $C^{p}$ candidate Lyapunov function $V_{g}: \mathbb{R}^{n+1} \rightarrow \mathbb{R}_{+}$as:

$$
V_{g}(y, x)=V_{x}(x)+(y-\mathscr{M}(x))^{\prime} P(y-\mathscr{M}(x)) .
$$

This function is proper and positive definite and, according to [3], it is a global weak CLF $^{5}$.

\footnotetext{
${ }^{5}$ Actually we can replace the function $\mathscr{M}$ by its first order approximation at the origin namely we can replace $\mathscr{M}(x)$ by $\frac{\partial \mathscr{M}}{\partial x}(0) x=M x$. But then the Lyapunov function in (35) has to be modified in

$$
V_{g}(y, x)=\ell\left(V_{x}(x)\right)+\left[\sqrt{1+(y-M x)^{\prime} P(y-M x)}-1\right]
$$

where $\ell$ is a $C^{1}$ class $\mathscr{K}^{\infty}$ function to be tuned large (enough). For more details, see [3], [13] or [12]. 
To complete Part 2 of the proof, it remains to show the quadratic approximation of the candidate Lyapunov function is $\mathscr{P}$. More precisely, it remains to show that $\mathscr{H}\left(V_{g}\right)(0,0)=2 \mathscr{P}$. Note that

$$
\mathscr{H}\left(V_{g}\right)(0,0)=\left[\begin{array}{cc}
2 P & -2 P \frac{\partial \mathscr{M}}{\partial x}(0) \\
-2\left(\frac{\partial \mathscr{M}}{\partial x}(0)\right)^{\prime} P & \mathscr{H}\left(V_{x}\right)(0)+2\left(\frac{\partial \mathscr{M}}{\partial x}(0)\right)^{\prime} \frac{\partial \mathscr{M}}{\partial x}(0)
\end{array}\right] .
$$

By evaluating the partial derivative of (34) at the origin where $f, \alpha_{x}$ and $h$ are zero, we get :

$$
\frac{\partial \mathscr{M}}{\partial x}(0)\left(F+G K_{x}\right)-A \frac{\partial \mathscr{M}}{\partial x}(0)=H .
$$

The eigenvalues of $\left(F+G K_{x}\right)$ and $A$ being all different, the solution of this algebraic equation is unique and with (19), we get:

$$
\frac{\partial \mathscr{M}}{\partial x}(0)=M=-P^{-1} Q
$$

Hence, equality (37) becomes:

$$
\mathscr{H}\left(V_{g}\right)(0,0)=2\left[\begin{array}{cc}
P & Q \\
Q^{\prime} & R
\end{array}\right]=2 \mathscr{P}
$$

\section{Part 3: Construction of the controller}

In this part of the proof we construct the global control law denoted $\alpha_{o}$ solution to stabilization with prescribed local behavior problem. This one is obtained by interpolating $K_{o}$ and a global control law $\alpha_{g}$.

By looking at the time derivative of $V_{g}$ along the solution of the system (2), we see that a control law ensuring stabilization of the origin of the system (2) and boundedness of the solutions can be obtained simply ${ }^{6}$ as:

$$
\alpha_{g}(y, x)=\alpha_{x}(x)-\frac{\partial V_{x}}{\partial x}(x) g(x)+2(y-\mathscr{M}(x))^{\prime} P \frac{\partial \mathscr{M}}{\partial x}(x) g(x) .
$$

Indeed, with (31) this gives along the trajectory of the system (2) :

$$
\left.\overbrace{V_{g}(y, x)}\right|_{u=\alpha_{g}(y, x)}=-W_{x}(x)-\left(\frac{\partial V_{x}}{\partial x}(x) G g(x)-2(y-\mathscr{M}(x))^{\prime} P \frac{\partial \mathscr{M}}{\partial x}(x) g(x)\right)^{2}
$$

\footnotetext{
${ }^{6}$ Note that to design this control, we need to construct the function $\mathscr{M}$ solution to the PDE (34). Hence, it may be difficult to apply this strategy for general feedforward systems. However, as shown in the following Section, when we consider some specific systems, this control law may be given in closed form. Moreover, when considering the Lyapunov function $V_{g}$ given in (36) and provided we are able to compute the function $\ell$, an explicit solution may be given.
} 
which is non positive. But, unfortunately, the first order approximation of the control law $\alpha_{g}$ is

$$
K_{g}=\left[\begin{array}{ll}
\frac{\partial \alpha_{g}}{\partial y}(0,0) & \frac{\partial \alpha_{g}}{\partial x}(0,0)
\end{array}\right]=\left[\begin{array}{ll}
K_{g, x} & K_{g, y}
\end{array}\right],
$$

with

$$
K_{g, x}=K_{x}-2 G^{\prime} R_{x}-2 G^{\prime} M^{\prime} P M, K_{g, y}=2 G^{\prime} M^{\prime} P .
$$

And this one is not equal to the given one $K_{o}$. Hence the control law $u=\alpha_{g}(y, x)$ is not a solution to the stabilization with prescribed local behavior.

The idea of the construction is to show that the two controllers $u=\alpha_{g}(y, x)$ and $u=K_{o, y} y+$ $K_{o, x} x$ makes the time derivative of a same Lyapunov function non positive in a small neighborhood of the origin.

Indeed, we have the following lemma whose proof is given in appendix.

Lemma 4 (Same Lyapunov function for the two controllers $\alpha_{g}$ and $K_{o}$ ): There exist a positive definite function $V_{L}: \mathbb{R}^{n_{y}} \times \mathbb{R}^{n_{x}} \rightarrow \mathbb{R}_{+}$and three positive real numbers $R_{L}, c_{g}$ and $c_{o}$ such that for all $(y, x)$ such that $V_{L}(y, x) \leq R_{L}$ we have

$$
\left.\overparen{V_{L}(y, x)}\right|_{u=\alpha_{g}(y, x)} \leq-c_{g}\left(|x|^{2}+\left|y^{\prime} P M G\right|^{2}\right)
$$

and,

$$
\left.\overparen{V_{L}(y, x)}\right|_{u=K_{o, y} y+K_{o, x} x} \leq-c_{o}\left[|x|^{2}+\left|y^{\prime} P M G\right|^{2}\right] .
$$

The proof of this Lemma relies on the use of change of coordinates which rectifies the controlled vector field $g$ around the origin. This property relies on Assumption 2.

The two controllers $K_{o}$ and $\alpha_{g}$ making non positive the time derivative of the same Lyapunov function and the system being input affine it yields that any convex combination of both controllers will have the same property. Hence, we can interpolate in a neighborhood of the origin this control law with the prescribed one.

$$
\alpha_{o}(y, x)=(1-\rho(y, x))\left(K_{o, y} y+K_{o, x} x\right)+\rho(y, x) \alpha_{g}(y, x)
$$

with $\rho$ any smooth function taking value in $[0,1]$ and such that:

$$
\rho(y, x)= \begin{cases}0 & \text { if } V_{L}(y, x) \leq \frac{1}{3} R_{L}, \\ 1 & \text { if } V_{L}(y, x) \geq \frac{2}{3} R_{L} .\end{cases}
$$


Indeed, in this case we get along the solution of the system (2) and for all $(y, x)$ such that $V_{L}(y, x) \leq R_{L}$

$$
\left.\overparen{V_{L}(y, x)}\right|_{u=\alpha_{o}(y, x)}=\left.(1-\rho(y, x)) \overparen{V_{L}(y, x)}\right|_{u=K_{o, y} y+K_{o, x} x}+\left.\rho(y, x) \overbrace{V_{L}(y, x)}\right|_{u=\alpha_{g}(y, x)} \leq 0 .
$$

This is sufficient to conclude that we have stability of the equilibrium. Note however that in order to study its asymptotic behavior and its convergence toward zero we make in the following section an analysis by introducing a Lyapunov function associated to our controller.

\section{Part 4: Modification of the global CLF to prove asymptotic stability}

In this part, we unite the two functions $V_{g}$ and $V_{L}$ in order to obtain a Lyapunov function associated to the controller $\alpha_{o}$. Following [14] consider the function $V_{o}$ defined as

$$
V_{o}(y, x)=\gamma\left(V_{L}(y, x)\right) V_{L}(y, x)+d\left[1-\gamma\left(V_{L}(y, x)\right)\right] V_{g}(y, x)
$$

where $\gamma$ is any smooth and non increasing function such that

$$
\gamma(s)= \begin{cases}0 & \text { if } s \geq R_{L} \\ 1 & \text { if } s \leq \frac{2}{3} R_{L}\end{cases}
$$

and $d$ is a positive real number such that

$$
V_{L}(y, x) \leq d V_{g}(y, x), \forall(y, x): \frac{2}{3} R_{L} \leq V_{L}(y, x) \leq R_{L} .
$$

Note that we have with $u=\alpha_{o}(y, x)$,

$$
\begin{aligned}
\left.\overparen{V_{o}(y, x)}\right|_{u=\alpha_{o}(y, x)}=\gamma^{\prime}\left(V_{L}(y, x)\right) & {\left.\left[V_{L}(y, x)-d V_{g}(y, x)\right] \overparen{V_{L}(y, x)}\right|_{u=\alpha_{o}(y, x)} } \\
& +\left.\gamma\left(V_{L}(y, x)\right) \overparen{V_{L}(y, x)}\right|_{u=\alpha_{o}(y, x)} ^{\cdot}+d\left(1-\left.\gamma\left(V_{L}(y, x)\right) \overparen{V_{g}(y, x)}\right|_{u=\alpha_{o}(y, x)} .\right.
\end{aligned}
$$

and different cases may be distinguished:

1) If $V_{L}(y, x) \geq R_{L}$. In this case, we have $\alpha_{o}(y, x)=\alpha_{g}(y, x), \gamma^{\prime}\left(V_{L}(y, x)\right)=0$ and $\gamma\left(V_{L}(y, x)\right)=$ 0 . Consequently, we get,

$$
\left.\overparen{V_{o}(y, x)}\right|_{u=\alpha_{o}(y, x)}=-d W_{x}(x)-d\left(\frac{\partial V_{x}}{\partial x}(x) G g(x)-2(y-\mathscr{M}(x))^{\prime} P \frac{\partial \mathscr{M}}{\partial x}(x) G g(x)\right)^{2},
$$

2) If $R_{L} \geq V_{L}(y, x) \geq \frac{2}{3} R_{L}$. In this case, we have $\alpha_{o}(y, x)=\alpha_{g}(y, x)$. Consequently, we have $\left.\overparen{V_{L}(y, x)}\right|_{u=\alpha_{o}(y, x)} \leq 0$ and $\left.\overparen{V_{g}(y, x)}\right|_{u=\alpha_{o}(y, x)} \leq 0$ Moreover we have $V_{L}(y, x)-d V_{g}(y, x) \leq 0$ 
and $\gamma^{\prime}\left(V_{L}(y, x)\right) \leq 0$. Hence, with (41) it yields,

$$
\begin{aligned}
& \left.\overparen{V_{o}(y, x)}\right|_{u=\alpha_{o}(y, x)} \leq-c_{g} \gamma\left(V_{L}(y, x)\right)\left[|x|^{2}+\left|y^{\prime} P M G\right|^{2}\right] \\
& -d\left(1-\gamma\left(V_{L}(y, x)\right)\left[W_{x}(x)+\left(\frac{\partial V_{x}}{\partial x}(x) G g(x)-2(y-\mathscr{M}(x))^{\prime} P \frac{\partial \mathscr{M}}{\partial x}(x) G g(x)\right)^{2}\right]\right.
\end{aligned}
$$

3) If $\frac{2}{3} R_{L} \geq V_{L}(y, x)$. We have $\gamma^{\prime}\left(V_{L}(y, x)\right)=0$ and $\gamma\left(V_{L}(y, x)\right)=1$. Hence, it yields,

$$
\left.\overparen{V_{o}(y, x)}\right|_{u=\alpha_{o}(y, x)}=\left.\gamma\left(V_{L}(y, x)\right) \overparen{V_{L}(y, x)}\right|_{u=\alpha_{o}(y, x)} .
$$

On another hand we have,

$$
\begin{aligned}
\left.\overparen{V_{L}(y, x)}\right|_{u=\alpha_{o}(y, x)} & =\left.(1-\rho(y, x)) \overparen{V_{L}(y, x)}\right|_{u=\left(K_{o, y} y+K_{o, x} x\right)}+\left.\rho(y, x) \overparen{V_{L}(y, x)}\right|_{\left.u=\alpha_{g}(y, x)\right)}, \\
& \leq-\left[c_{o}(1-\rho(y, x))+c_{g} \rho(y, x)\right]\left[|x|^{2}+\left|y^{\prime} M G\right|^{2}\right] .
\end{aligned}
$$

Hence, we get global stability and local asymptotic stabilization. Moreover, the control law satisfies the local property requested.

Finally, from LaSalle invariance principle, it follows that, for each trajectory, there exists a real number $v$ such that it converges the trajectories converge to the largest set of points $(y, 0)$, invariant for the system

$$
\dot{y}=A y
$$

and satisfying

$$
V_{o}(y, 0)=v \quad, \quad y^{\prime} P M G=0
$$

Recalling that $M=-P^{-1} Q$, we get the result.

\section{ILLUSTRATION ON A PARTICULAR CLASS OF SYSTEMS}

In this section, we consider the problem of designing a robust stabilizing control law for a class of disturbed strict feedforward systems with linear $x$ dynamics and a quadratic function $h$. In other words we consider the case in which the system (2) is in the form

$$
\left\{\begin{array}{l}
\dot{y}=A y+h(x)+d_{y}(y, x) \omega \\
\dot{x}=F x+G u+d_{x}(y, x) \omega
\end{array},\right.
$$

where to simplify the presentation we consider the mono input case (i.e. $m=1$ ) and $\omega$ is an unknown input which is assumed to be a locally bounded time function taking values in $\mathbb{R}^{n_{\omega}}$ and 
$d_{y}$ and $d_{x}$ are locally Lipschitz function of appropriate dimension. We assume that the function $h(x)$ is a quadratic function. Hence, this one can be written in the form

$$
h(x)=H x+\left[\begin{array}{c}
x^{\prime} H_{1} x \\
\vdots \\
x^{\prime} H_{n_{y}} x
\end{array}\right],
$$

where for all $i$ in $\left\{1, \cdots, n_{y}\right\}, H_{i}^{\prime}=H_{i}$ is in $\mathbb{R}^{n_{x} \times n_{x}}$.

A framework to design a robust control law for this system can be to follow the $H_{\infty}$ design methodology (see [15]). In this context, we are looking for a control law that satisfies two distinct objectives:

1) The first one is to guarantee the asymptotic stability of the origin when the disturbance vanishes.

2) The second one is to guarantee a given attenuation level of a quadratic functional of the state and control in the $L_{2}$ framework. More precisely, given a positive definite matrix $Q$ in $\mathbb{R}^{\left(n_{x}+n_{y}\right) \times\left(n_{x}+n_{y}\right)}$ and a positive real number $\gamma$ (the attenuation level) we want to find a stabilizing feedback control law $u=\alpha_{o}(y, x)$ such that the following inequality is satisfied for all $t$ in $\mathbb{R}_{+}$:

$$
\int_{0}^{t}\left[(y(s), x(s))^{\prime} \mathrm{Q}(y(s), x(s))+u(s)^{2}\right] d s \leq \gamma^{2} \int_{0}^{t}|\omega(s)|^{2} d s,
$$

where $(y(),. x()$.$) denotes the solution of system (44) initialized to the origin.$

Solving this problem relies on the construction of a solution to a nonlinear Hamilton Jacobi Bellman equality which can be difficult (or impossible) to solve (see [15]).

However, if we focuss on the linear approximation of system (44), then this problem can be solved locally. The first order approximation of system (44) is a linear system defined as:

$$
\left\{\begin{array}{l}
\dot{y}=A y+H x+D_{y} \omega \\
\dot{x}=F x+G u+D_{x} \omega
\end{array} \quad, D_{y}=d_{y}(0,0), D_{x}=d_{x}(0,0) .\right.
$$

In compact form, this linear system can be rewritten as follows.

$$
\dot{\chi}=\mathscr{A}_{0} \chi+\mathscr{G} u+\mathscr{D} \omega \quad \chi^{\prime}=\left[y^{\prime}, x^{\prime}\right] .
$$

In the linear context, the Hamilton Jacobi Bellman equality is an algebraic equation defined as:

$$
\mathscr{S} \mathscr{A}_{0}+\mathscr{A}_{0}^{\prime} \mathscr{S}+\frac{1}{\gamma} \mathscr{S} \mathscr{D} \mathscr{D}^{\prime} \mathscr{S}-\mathscr{S} \mathscr{G} \mathscr{G}^{\prime} \mathscr{S}+\mathrm{Q}=0
$$


where the solution $\mathscr{S}$ is a positive definite matrix in $\mathbb{R}^{\left(n_{x}+n_{y}\right) \times\left(n_{x}+n_{y}\right)}$, and a robust linear control for system (47) solving the disturbance attenuation problem as defined by inequality (46) for the linear approximation is given as:

$$
u=K_{o}[y, x]^{\prime}=-\mathscr{G}^{\prime} \mathscr{S} \chi .
$$

However, this control law guarantees only local asymptotic stability of the origin of system (44). We may apply the design methodology given in Theorem 1 to design a global asymptotic stabilizing controller $u=\alpha_{o}(y, x)$ such that its local behavior is exactly $K_{o}$.

We assume that the system (47) is controllable and the matrix $A$ is skew symmetric. This yields that Assumption 1 and 3 are satisfied. Moreover, the $x$ subsystem being linear, it yields that Assumption 2 and 4 are trivially satisfied. In this case, we may apply the procedure of Theorem 1 as described by the following four steps.

1) First, we choose a local prescribed behavior $K_{o}$ by solving the HJB algebraic equation (48) for given tuning parameters $Q$ and $\gamma$.

2) We solve the linear matrix inequality (14) to find a forwarding like matrix $\mathscr{P}$ (see Theorem 1 equation (10)). Note that this step is not guaranteed to succeed when $n_{y}>1$. This gives us a stabilizing controller $K_{x}$ for the $x$-subsystem (see equation (28)) and its associated Lyapunov matrix $R_{x}$ (given in equation (18)).

3) Then, using this $K_{x}$ and $R_{x}$, we give an explicit solution $\mathscr{M}$ to the partial differential equation (34). As shown in appendix D, in our particular context this one can simply be expressed as

$$
\mathscr{M}(x)=M x+\left[\begin{array}{c}
x^{\prime} M_{1} x \\
\vdots \\
x^{\prime} M_{n_{y}} x
\end{array}\right],
$$

where $M$ is solution to the Sylvester equation

$$
M\left[F+G K_{x}\right]-A M=H,
$$

and $\left(M_{1}, \cdots, M_{n_{y}}\right)$ are matrices in $\mathbb{R}^{n_{x} \times n_{x}}$ obtained by solving the unsquare Sylvester 
equation ${ }^{7}$ :

$$
\left[I_{n_{y}} \otimes\left[F+G K_{x}\right]^{\prime}-A \otimes I_{n_{x}}\right] \cdot\left[\begin{array}{c}
M_{1} \\
\vdots \\
M_{n_{y}}
\end{array}\right]+\left[\begin{array}{c}
M_{1} \\
\vdots \\
M_{n_{y}}
\end{array}\right] \cdot\left[F+G K_{x}\right]=\left[\begin{array}{c}
H_{1} \\
\vdots \\
H_{n_{y}}
\end{array}\right],
$$

where the operator $\otimes$ is the Kronecker tensor product.

4) Next we construct the globally stabilizing feedback law defined as

$$
\alpha_{g}(y, x)=\left(K_{x}-2 G^{\prime} R_{x}\right) x+2[y-\mathscr{M}(x)]^{\prime} P \frac{\partial \mathscr{M}}{\partial x}(x) G .
$$

and we modify this, control law to match the local desired behavior. To complete the modified forwarding procedure, we construct a control law $\alpha_{o}(y, x)$ (see equation (43)) with function $\rho(y, x)$ chosen as :

$$
\rho(y, x)= \begin{cases}0 & \text { if }\left[y^{\prime} x^{\prime}\right] \mathscr{P}\left[\begin{array}{l}
y \\
x
\end{array}\right] \leq \frac{1}{3} R_{L}, \\
\frac{3}{R_{l}}\left[y^{\prime} x^{\prime}\right] \mathscr{P}\left[\begin{array}{l}
y \\
x
\end{array}\right]-1 & \text { if } \frac{1}{3} R_{L} \leq\left[y^{\prime} x^{\prime}\right] \mathscr{P}\left[\begin{array}{l}
y \\
x
\end{array}\right] \leq \frac{2}{3} R_{L}, \\
1 & \text { if }\left[y^{\prime} x^{\prime}\right] \mathscr{P}\left[\begin{array}{l}
y \\
x
\end{array}\right] \geq \frac{2}{3} R_{L} .\end{cases}
$$

where $R_{L}$ is computed with Lemma 4.

The benchmark example [9, equation (39)] (see also [10]) fits in the class of system considered in this section. More precisely, system (44) is studied in the particular cases in which $n_{y}=1$, $n_{x}=2$ and the parameters are selected as follows.

$$
A=0, H_{1}=\left[\begin{array}{cc}
1 & -1 \\
-1 & 1
\end{array}\right], H=\left[\begin{array}{ll}
1 & 0
\end{array}\right], F=\left[\begin{array}{ll}
0 & 1 \\
0 & 0
\end{array}\right], G=D_{x}=\left[\begin{array}{l}
0 \\
1
\end{array}\right], D_{y}=0 .
$$

For this system we can follow the procedure to design a global stabilizer with local optimality.

1) We select the tuning parameter of the local optimal controller as:

$$
\mathrm{Q}=\left[\begin{array}{ccc}
1 & 0 & 0 \\
0 & 1 & 0 \\
0 & 0 & 10
\end{array}\right], \gamma \approx 1.00
$$

Solving the associated Riccati equation (see equation (48)) by employing the routine (care) of Matlab with the attenuation level $\gamma \approx 1.00$, it yields the local optimal controller

$$
K_{o} \approx\left[\begin{array}{lll}
-4.3674 & -26.9105 & -80.7232
\end{array}\right] \text {. }
$$

\footnotetext{
${ }^{7}$ It is shown in appendix D that this Sylvester equation admits a solution.
} 
2) In the case $n_{y}=1$, we obtain directly the matrix $\mathscr{P}$ given as

$$
\mathscr{P} \approx\left[\begin{array}{lll}
1 & 3 & 4.37 \\
3 & 14.11 & 29.91 \\
4.37 & 29.91 & 80.72
\end{array}\right] .
$$

This yields the controller $K_{x}$ and the matrix $R_{x}$ given as:

$$
K_{x} \approx\left[\begin{array}{ll}
-2.9997 & -4.36
\end{array}\right], R_{x} \approx\left[\begin{array}{ll}
-3.39 & -2.18 \\
-2.18 & -3.90
\end{array}\right] .
$$

3) We get the solution to the PDE given in equation (50) with

$$
M \approx\left[\begin{array}{ll}
-2.11 & -2.16
\end{array}\right], M_{1} \approx\left[\begin{array}{ll}
-5.61 & -2.16 \\
-2.16 & -3.24
\end{array}\right] .
$$

4) By a Matlab computation, it yields the positive real number $R_{L}$ given as:

$$
R_{L} \approx 5 .
$$

In the following figures is considered simulation of this control law when considering $\omega$ to be a gaussian white noise with variance 2 .

In Figure 1 is shown a state trajectory when considering a particular initial condition for the disturbed model.

Fig. 1. Example of a closed loop trajectory

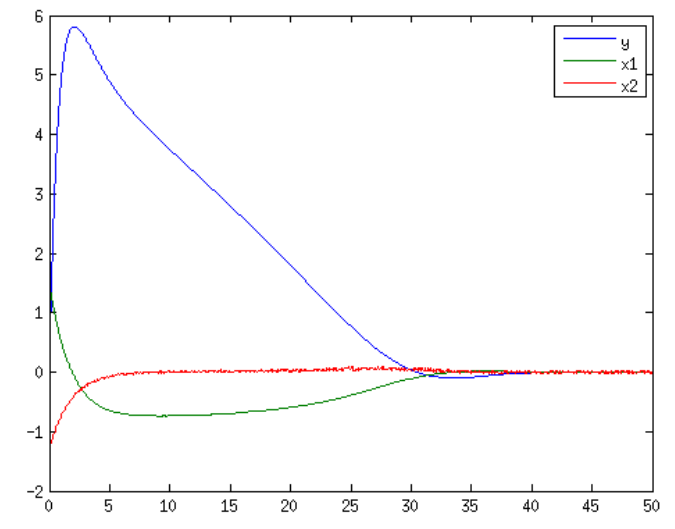

In Figure 2 is depicted the associated control law. The red one is the locally optimal control. The blue is the evaluation of the optimal local and linear control law along the solution of the 
system. Finally the green one is the evaluation of the global forwarding control law. It can be checked that the solution goes from the green toward the red one when the solution gets close to the origin.

Fig. 2. Controllers

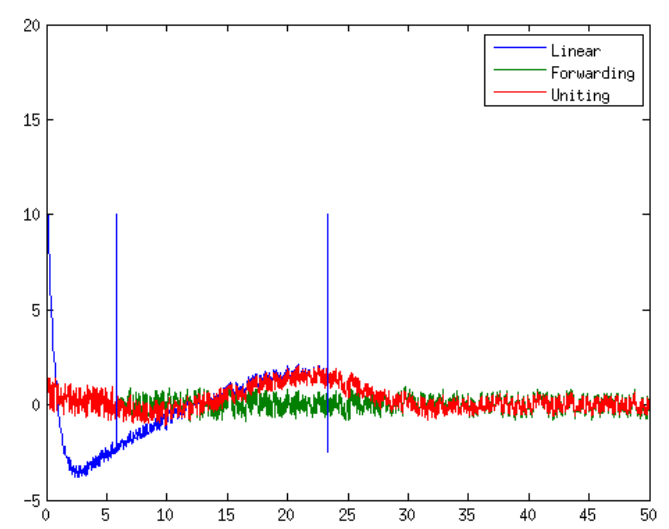

The last figure compares the proposed control law which is locally optimal with respect to a given cost and the control law given in [9, equation (39)] when considering solution initiated from the origin.

Fig. 3. Comparison with the controller of [9, equation (39)].

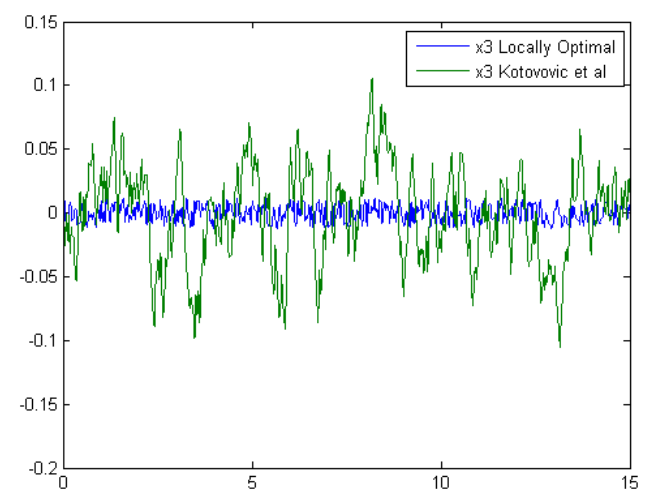

\section{CONCLUSION}

We have studied the problem of designing a stabilizing controller which ensures a desired local behavior. We have shown that given a prescribed locally stabilizing control law, provided 
there exists a Lyapunov matrix with a specific structure, this stabilizing local behaviors can be reproduced when using the forwarding design technique developed in [3], [4]. This is made possible by modifying the forwarding design adequately. Note that when the $y$ subsystem is of dimension 1, this result establishes that all stabilizing local behaviors can be reproduced. This result gives a theoretical justification of a statistical result given in [8].

\section{APPENDIX}

A. On the feasibility of the weak Lyapunov inequality

In this Section, we study the feasibility of the weak Lyapunov inequality (10) when considering systems whose first order approximation is the system (15). Assume a stabilizing local controller $K_{o}=\left[0, k_{2}, k_{x}\right]$ is given. Note that, the necessary and sufficient conditions to make $u=k_{2} y_{2}+k_{x} x$ a globally and asymptotically stabilizing input are

$$
k_{2}<0, k_{x}<0 \text {. }
$$

First of all note that $A=\left[\begin{array}{cc}0 & -1 \\ 1 & 0\end{array}\right]$. Let $P=\left[\begin{array}{ll}P_{11} & P_{12} \\ P_{12} & P_{22}\end{array}\right]$ in $\mathbb{R}^{2 \times 2}$ be a Lyapunov matrix which satisfies (5). Note that

$$
P A+A^{\prime} P=\left[\begin{array}{cc}
2 P_{12} & P_{22}-P_{11} \\
P_{22}-P_{11} & -2 P_{12}
\end{array}\right]=0 \text {. }
$$

Hence, this implies that $P_{12}=0$ and $P_{22}=P_{11}$. In other word, the only Lyapunov matrix which satisfies (5) are those which take the form $P=p I_{2}$. For a candidate Lyapunov matrix in the form (we have normalized with respect to $p$ ):

$$
\mathscr{P} \triangleq\left(\begin{array}{ccc}
1 & 0 & q_{1} \\
0 & 1 & q_{2} \\
q_{1} & q_{2} & r
\end{array}\right)
$$

to, be positive definite, parameters $q_{1}, q_{2}$ and $r$ must satisfy:

$$
r>q_{1}^{2}+q_{2}^{2}
$$

Assume there exists $k_{2}$ and $k_{x}$ which satisfies (56) and such that there exists $q_{1}, q_{2}$ and $r$ with $q_{1}$ and $q_{2}$ not equal to zero such that the weak Lyapunov inequality (10) is satisfied. Inequality 
(10) implies that the following matrix is non positive

$$
\mathrm{Q} \triangleq\left[\mathscr{A}^{\prime} \mathscr{P}+\mathscr{P} \mathscr{A}^{\prime}\right]=\left[\begin{array}{ccc}
0 & k_{2} q_{1} & q_{2}+k_{x} q_{1} \\
* & 2 k_{2} q_{2} & -q_{1}+k_{2} r+1+k_{x} q_{2} \\
* & * & 2 q_{2}+2 k_{x} r
\end{array}\right] .
$$

It implies that for all $\left(v_{1}, v_{2}\right)$ in $\mathbb{R}^{2}$, we have

$$
\left[v_{1}, v_{2}, 0\right] \mathrm{Q}\left[\begin{array}{c}
v_{1} \\
v_{2} \\
0
\end{array}\right] \leq 0 \Leftrightarrow k_{2} q_{1} v_{2} v_{1}-2 k_{2} q_{2} v_{2}^{2} \leq 0 .
$$

Hence, this implies that $q_{1}=0$. Moreover for all $\left(v_{1}, v_{3}\right)$ in $\mathbb{R}^{2}$, we have

$$
\left[v_{1}, 0, v_{3}\right] \mathrm{Q}\left[\begin{array}{c}
v_{1} \\
0 \\
v_{3}
\end{array}\right]:=\left[v_{1}, 0, v_{3}\right]\left[\begin{array}{ccc}
0 & 0 & q_{2} \\
* & 2 k_{2} q_{2} & k_{2} r+1+k_{x} q_{2} \\
* & * & 2 q_{2}+2 k_{x} r
\end{array}\right]\left[\begin{array}{c}
v_{1} \\
0 \\
v_{3}
\end{array}\right] \leq 0,
$$

which means for all $\left(v_{1}, v_{3}\right)$ in $\mathbb{R}^{2}$

$$
q_{2} v_{3} v_{1}+\left(2 q_{2}+2 k_{x} r\right) v_{3}^{2} \leq 0 .
$$

Hence, $q_{1}=q_{2}=0$ which contradicts the assertion.

\section{B. proof of Lemma 2}

The proof of this Lemma is based on recent results obtained in [14]. Indeed, the design of the function $V_{x}$ is obtained from the uniting of a quadratic local control Lyapunov function (denoted $V_{0}$ ) and a global control Lyapunov function (denoted $V_{\infty}$ ) obtained employing a converse Lyapunov theorem.

First of all, employing the converse Lyapunov theorem of Kurzweil [16], there exists a $C^{\infty}$ function $V_{\infty}: \mathbb{R}^{n} \rightarrow \mathbb{R}_{+}$such that

$$
\frac{\partial V_{\infty}}{\partial x}(x)\left[f(x)+g(x) \alpha_{x}(x)\right]<0, \forall x \neq 0 .
$$

On the other hand, with (20), the function $V_{0}(x)=x^{\prime} R_{x} x$ is such that,

$$
\frac{\partial V_{0}}{\partial x}(x)\left[F+G K_{x}\right] x<0, \forall x \neq 0 .
$$


Due to the fact that $K_{x}$ satisfies equation (7) it yields that the matrix $F+G K_{x}$ is the first order approximation of the $x$-subsystem in equation (2) with the control law $u=\alpha_{x}(x)$. Consequently, it implies that there exists a positive real number $\varepsilon_{1}$ such that

$$
\frac{\partial V_{0}}{\partial x}(x)\left[f(x)+g(x) \alpha_{x}(x)\right]<0, \forall|x| \leq \varepsilon_{1} .
$$

Employing [14, Theorem 2.1], it yields the existence of a function $V_{x}$ and a positive real number $\varepsilon_{2}$ such that

1) for all $x$ in $\mathbb{R}^{n} \backslash\{0\}$,

$$
\frac{\partial V_{x}}{\partial x}(x)\left[f(x)+g(x) \alpha_{x}(x)\right]<0 .
$$

2) for all $x$ in $\mathbb{R}^{n}$ such that $|x| \leq \varepsilon_{2}$, we have

$$
V_{x}(x)=V_{0}(x),
$$

and consequently $\mathscr{H}\left(V_{x}\right)(0)=2 R_{x}$.

This conclude the proof of Lemma 2

\section{Proof of Lemma 4}

With Assumption 2, there exists a neighborhood of the origin in which the distribution Vect $\left\{g_{1}(x), \ldots, g_{m}(x)\right\}$ is regular, involutive and of constant dimension $m$. Employing Frobenius theorem [17, Theorem 1.4.1], we know there exists a neighborhood of the origin $U \subset \mathbb{R}^{n_{x}}$ and a diffeomorphism $\varphi=\left(\varphi_{1}, \ldots, \varphi_{n_{x}}\right): U \rightarrow \varphi(U)$ such that

$$
\frac{\partial \varphi_{i}}{\partial x}(x) g_{j}(x)=0, m+1 \leq i \leq n_{x}, 1 \leq j \leq m, \forall x \in U .
$$

Let $\psi: U \rightarrow \psi(U)$ be the diffeomorphism defined as $\left(\frac{\partial \varphi}{\partial x}(0)\right)^{-1} \varphi(x)$. Note that we have $\frac{\partial \psi}{\partial x}(0)=$ $I_{n_{x}}$. Moreover, we have

Hence, we get

$$
G=g(0)=\left(\frac{\partial \varphi}{\partial x}(0)\right)^{-1}\left[\begin{array}{c}
\frac{\partial \varphi_{1}}{\partial x}(0) g(0) \\
\vdots \\
\frac{\partial \varphi_{m}}{\partial x}(0) g(0) \\
0 \\
\vdots
\end{array}\right]
$$

$$
\frac{\partial \psi}{\partial x}(x) g(x)=G r(x), \forall x \in U
$$


where

$$
r(x)=\left[\begin{array}{c}
\frac{\partial \varphi_{1}}{\partial x}(0) g(0) \\
\vdots \\
\frac{\partial \varphi_{m}}{\partial x}(0) g(0)
\end{array}\right]^{-1}\left[\begin{array}{c}
\frac{\partial \varphi_{1}}{\partial x}(x) g(x) \\
\vdots \\
\frac{\partial \varphi_{m}}{\partial x}(x) g(x)
\end{array}\right]
$$

If we denote the new coordinates $\tilde{x}=\psi(x)$, the system (2) takes the form

$$
\dot{\tilde{x}}=\tilde{f}(\tilde{x})+G \tilde{r}(\tilde{x}) u
$$

where $\tilde{r}(\tilde{x})$ is a matrix in $\mathbb{R}^{m \times m}$ for all $\tilde{x}$ in $\psi(U)$ and $\tilde{r}(0)=I_{m}$. Note that since $\frac{\partial \psi}{\partial x}(0)=I_{n_{x}}$, we have $\frac{\partial \tilde{f}}{\partial \tilde{x}}(0)=F$.

Consider now the Lyapunov function

$$
V_{L}(y, x)=\left[\begin{array}{ll}
y^{\prime} & \psi(x)^{\prime}
\end{array}\right] \mathscr{P}\left[\begin{array}{c}
y \\
\psi(x)
\end{array}\right]
$$

We will show that this Lyapunov function is nonincreasing in a neighborhood of the origin when employing the control law $u=\alpha_{g}(y, x)$. Note that there exists $a$ and $b$ such that

$$
\alpha_{g}(y, x)=a(\tilde{x})+b(\tilde{x}) y
$$

Note that we have the properties

$$
\frac{\partial a}{\partial \tilde{x}}(0)=K_{g, x}, b(0)=K_{g, y}
$$

When $u=\alpha_{g}(y, x)$ the time derivative of the function $V_{L}(y, x)$ satisfies along the trajectories of the nonlinear system (2)

$$
\left.\overbrace{V_{L}(y, x)}\right|_{u=\alpha_{g}(y, x)}=2\left[\begin{array}{ll}
y^{\prime} & \tilde{x}^{\prime}
\end{array}\right] \mathscr{P}\left[\begin{array}{c}
A y+\tilde{h}(\tilde{x}) \\
\tilde{f}(\tilde{x})+G \tilde{r}(\tilde{x}) \alpha_{g}(y, x)
\end{array}\right] .
$$

This gives

$$
\begin{aligned}
& \left.\overparen{V_{L}(y, x)}\right|_{u=\alpha_{g}(y, x)}=2\left[\begin{array}{ll}
y^{\prime} & \tilde{x}^{\prime}
\end{array}\right] \mathscr{P}\left(\left[\begin{array}{cc}
A & H \\
G K_{g, y} & F+G K_{g, x}
\end{array}\right]\left[\begin{array}{l}
y \\
\tilde{x}
\end{array}\right]\right.
\end{aligned}
$$

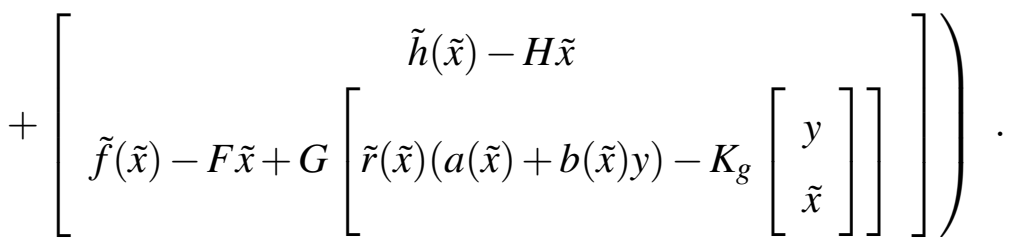


Hence we get,

$$
\left.\overbrace{V_{L}(y, x)}^{\cdot}\right|_{u=\alpha_{g}(y, x)}=\left[\begin{array}{ll}
y^{\prime} Q G & \tilde{x}^{\prime}
\end{array}\right] \mathbb{M}\left[\begin{array}{c}
G^{\prime} Q^{\prime} y \\
\tilde{x}
\end{array}\right]+\Delta_{1}(\tilde{x})+y^{\prime} \Delta_{2}(\tilde{x})+y^{\prime} Q G \Delta_{3}(\tilde{x}) y
$$

where,

$$
\mathbb{M}=\left[\begin{array}{cc}
-4 & -4\left(G^{\prime} M^{\prime} P M+G^{\prime} R_{x}\right) \\
-4\left(R_{x} G+M^{\prime} P M G\right) & S_{x}-4\left(G^{\prime} M^{\prime} P M+R_{x} G^{\prime}\right)\left(G R_{x}+M^{\prime} P M G\right)
\end{array}\right]<-c_{\mathbb{M}} I_{n_{x}+1}
$$

where $S_{x}$ is the negative definite matrix defined in (30) and $c_{\mathbb{M}}=\lambda_{\max }\{\mathbb{M}\}$ is a positive real number,

$\Delta_{1}(\tilde{x})=2\left[\begin{array}{ll}0 & \tilde{x}^{\prime}\end{array}\right] \mathscr{P}\left[\begin{array}{c}\tilde{h}(\tilde{x})-H \tilde{x} \\ \tilde{f}(\tilde{x})-F \tilde{x}+G\left[\tilde{r}(\tilde{x}) a(\tilde{x})-K_{g, x} \tilde{x}\right]\end{array}\right]$

$\Delta_{2}(\tilde{x})=2\left[\begin{array}{ll}1 & 0\end{array}\right] \mathscr{P}\left[\begin{array}{c}\tilde{h}(\tilde{x})-H \tilde{x} \\ \tilde{f}(\tilde{x})-F \tilde{x}+G\left[\tilde{r}(\tilde{x}) a(\tilde{x})-K_{g, x} \tilde{x}\right]\end{array}\right]+\left(\left[\begin{array}{ll}0 & \tilde{x}^{\prime}\end{array}\right] \mathscr{P}\left[\begin{array}{c}0 \\ G\left[\tilde{r}(\tilde{x}) b(\tilde{x})-K_{g, y}\right]\end{array}\right]\right)^{\prime}$

$\Delta_{3}(\tilde{x})=2 \tilde{r}(\tilde{x}) b(\tilde{x})-K_{g, y}$

Moreover, we have,

$$
\left|y^{\prime} Q G \Delta_{3}(\tilde{x}) y\right| \leq \frac{\left|y^{\prime} Q G\right|^{2}+\left|\Delta_{3}(\tilde{x})\right|^{2}}{2}|y|
$$

Hence, (58) becomes,

$$
\left.\overparen{V_{L}(y, x)}\right|_{u=\alpha_{g}(y, x)} \leq-|\tilde{x}|^{2}\left(c_{\mathbb{M}}-\frac{\Delta_{1}(\tilde{x})}{|\tilde{x}|^{2}}-|y| \frac{\left|\Delta_{2}(\tilde{x})\right|+\frac{1}{2}\left|\Delta_{3}(\tilde{x})\right|^{2}}{|\tilde{x}|^{2}}\right)-\left|G^{\prime} Q^{\prime} y\right|^{2}\left(c_{\mathbb{M}}-\frac{1}{2}|y|\right)
$$

Note that

$$
\left|\Delta_{1}(\tilde{x})\right|=O\left(|\tilde{x}|^{3}\right),\left|\Delta_{2}(\tilde{x})\right|=O\left(|\tilde{x}|^{2}\right),\left|\Delta_{3}(\tilde{x})\right|=O(|\tilde{x}|) .
$$

Hence, this implies that there exists a positive real number $R_{g}$ such that when $u=\alpha_{g}(y, x)$ we get along the solutions of the system (2)

$$
\left.\overparen{V_{L}(y, x)}\right|_{u=\alpha_{g}(y, x)} \leq-\frac{c_{\mathbb{M}}}{2}\left[|\tilde{x}|^{2}+\left|y^{\prime} P M G\right|^{2}\right], \forall(x, y): V_{L}(y, x) \leq R_{g} .
$$

With the same analysis, it is possible to find a positive real number $R_{l}$ such that by taking $u=K_{o, y} y+K_{o, x} x$ we get a positive real number $c_{o}$ such along the trajectories of the closed loop system we have

$$
\left.\overparen{V_{L}(y, x)}\right|_{u=K_{o, y} y+K_{o, x} x} \leq-c_{o}\left[|\tilde{x}|^{2}+\left|y^{\prime} P M G\right|^{2}\right], \forall(x, y): V_{L}(y, x) \leq R_{l} .
$$

Note that since $\psi$ is a diffeomorphism, we get the existence of a positive real number such $c_{\psi}$ that around the origin $|\tilde{x}| \geq c_{\psi}|x|$. Hence, we get the result with $R_{L}=\min \left\{R_{l}, R_{g}\right\}$. 
D. Solving the PDE for the illustrative example

In this section we show that given, a vector $K_{x} \in \mathbb{R}^{n_{x}}$ such that $F+G K_{x}$ is Hurwitz the following partial differential equation

$$
\frac{\partial \mathscr{M}}{\partial x}(x)\left[F+G K_{x}\right] x=A \mathscr{M}(x)+h(x), \forall x \in \mathbb{R}^{n} .
$$

can be solved explicitly when the function $h$ is the quadratic function defined in (45).

First of all, note that following (33), the solution $\mathscr{M}: \mathbb{R}^{n_{x}} \rightarrow \mathbb{R}^{n_{y}}$ can be expressed as

$$
\mathscr{M}(x)=\int_{+\infty}^{0} \exp (-A s) h\left(\exp \left(\left[F+G K_{x}\right] s\right) x\right) d s
$$

The function $h$ being quadratic, this implies that the function $\mathscr{M}$ is also quadratic and may be written in the form (50) with $\left(M, M_{1}, \ldots, M_{n_{y}}\right)$ are matrices in $\mathbb{R}^{n_{x} \times n_{x}}$ to be selected. Assume for the time being that there exist solutions to the two Sylvester equations (51) and (52) and let $\left(M, M_{1}, \ldots, M_{n_{y}}\right)$ be these solutions. Note that we have,

$$
\frac{\partial \mathscr{M}}{\partial x}(x)\left[F+G K_{x}\right] x=\left[I_{n_{y}} \otimes x^{\prime}\right] \Psi x+M\left[F+G K_{x}\right] x,
$$

where $\Psi$ is the matrix in $\mathbb{R}^{\left(n_{y} n_{x}\right) \times n_{x}}$ defined by

$$
\Psi=\left[\begin{array}{c}
M_{1}\left(F+G K_{x}\right)+\left(F+G K_{x}\right)^{\prime} M_{1} \\
\vdots \\
M_{n_{y}}\left(F+G K_{x}\right)+\left(F+G K_{x}\right)^{\prime} M_{n_{y}}
\end{array}\right] .
$$

However, employing the fact that $\left[M_{1}, \ldots, M_{n_{y}}\right]^{\prime}$ is solution to the Sylvester equation (52), we get

$\Psi=\left[I_{n_{y}} \otimes\left[F+G K_{x}\right]^{\prime}\right]\left[\begin{array}{c}M_{1} \\ \vdots \\ M_{n_{y}}\end{array}\right]+\left[\begin{array}{c}M_{1} \\ \vdots \\ M_{n_{y}}\end{array}\right]\left[F+G K_{x}\right]+\left[\begin{array}{c}H_{1} \\ \vdots \\ H_{n_{y}}\end{array}\right]=\left[A \otimes I_{n_{x}}\right]\left[\begin{array}{c}M_{1} \\ \vdots \\ M_{n_{y}}\end{array}\right]+\left[\begin{array}{c}H_{1} \\ \vdots \\ H_{n_{y}}\end{array}\right]$.

Hence, it implies

$$
\frac{\partial \mathscr{M}}{\partial x}(x)\left[F+G K_{x}\right] x=\left[I_{n_{y}} \otimes x^{\prime}\right]\left[A \otimes I_{n_{x}}\right]\left[\begin{array}{c}
M_{1} \\
\vdots \\
M_{n_{y}}
\end{array}\right] x+\left[I_{n_{y}} \otimes x^{\prime}\right]\left[\begin{array}{c}
H_{1} \\
\vdots \\
H_{n_{y}}
\end{array}\right] x+A M x+H x
$$


where we have used the fact that $M$ satisfies the Sylvester equation (51). Employing the fact that

$$
\left[I_{n_{y}} \otimes x^{\prime}\right]\left[A \otimes I_{n_{x}}\right]\left[\begin{array}{c}
M_{1} \\
\vdots \\
M_{n_{y}}
\end{array}\right]=A\left[\begin{array}{c}
x^{\prime} M_{1} \\
\vdots \\
x^{\prime} M_{n_{y}}
\end{array}\right]
$$

it yields

$$
\frac{\partial \mathscr{M}}{\partial x}(x)\left[F+G K_{x}\right] x=A\left[\begin{array}{c}
x^{\prime} M_{1} \\
\vdots \\
x^{\prime} M_{n_{y}}
\end{array}\right] x+\left[I_{n_{y}} \otimes x^{\prime}\right]\left[\begin{array}{c}
H_{1} \\
\vdots \\
H_{n_{y}}
\end{array}\right] x+A M x+H x=A \mathscr{M}(x)+h(x) .
$$

Consequently, if $\left(M, M_{1}, \ldots, M_{n_{y}}\right)$ are matrices in $\mathbb{R}^{n_{x} \times n_{x}}$ solution to the Sylvester equations (51) and (52) the function $\mathscr{M}$ defined in (50) is solution to the PDE (64).

It remains to show that the two Sylvester equations (51) and (52) admit a solution. Note that for the first one (i.e. equation (51)), this is trivial since the two matrices $F+G K_{x}$ and $-A$ have different eigenvalues $\left(F+G K_{x}\right.$ is Hurwitz and $A$ is stable).

The same property holds for equation (52). Indeed, with the matrix $R_{x}$ given in equation (18), we have

$$
\begin{aligned}
&\left(I_{n_{y}} \otimes R_{x}\right)\left[I_{n_{y}} \otimes\left[F+G K_{x}\right]-A^{\prime} \otimes I_{n_{x}}\right]+\left[I_{n_{y}} \otimes\left[F+G K_{x}\right]^{\prime}-A \otimes I_{n_{x}}\right]\left(I_{n_{y}} \otimes R_{x}\right) \\
&=I_{n_{y}} \otimes\left(R_{x}\left[F+G K_{x}\right]+\left[F+G K_{x}\right]^{\prime} R_{x}\right)-\left(I_{n_{y}} \otimes R_{x}\right)\left(A^{\prime} \otimes I_{n_{x}}\right)-\left(A \otimes I_{n_{x}}\right)\left(I_{n_{y}} \otimes R_{x}\right)
\end{aligned}
$$

Note that we have,

$$
\left(I_{n_{y}} \otimes R_{x}\right)\left(A^{\prime} \otimes I_{n_{x}}\right)+\left(A \otimes I_{n_{x}}\right)\left(I_{n_{y}} \otimes R_{x}\right)=A^{\prime} \otimes R_{x}+A \otimes R_{x}=0
$$

since, by assumption, $A^{\prime}=-A$. Consequently, it implies with (20)

$$
\begin{aligned}
\left(I_{n_{y}} \otimes R_{x}\right)\left[I_{n_{y}} \otimes\left[F+G K_{x}\right]-A^{\prime} \otimes I_{n_{x}}\right]+\left[I_{n_{y}} \otimes\left[F+G K_{x}\right]^{\prime}-A \otimes I_{n_{x}}\right]\left(I_{n_{y}} \otimes R_{x}\right) & \\
& =I_{n_{y}} \otimes\left(R_{x}\left[F+G K_{x}\right]+\left[F+G K_{x}\right]^{\prime} R_{x}\right)<0
\end{aligned}
$$

which is a Lyapunov equality with Lyapunov matrix $\left(I_{n_{y}} \otimes R_{x}\right)$. Hence, it establishes that the matrix $\left[I_{n_{y}} \otimes\left[F+G K_{x}\right]-A^{\prime} \otimes I_{n_{x}}\right]$ is Hurwitz and has different eigenvalues with the matrix $-\left[F+G K_{x}\right]$. 


\section{REFERENCES}

[1] M. Krstic, I. Kanellakopoulos, and P. Kokotovic, Nonlinear and Adaptive Control Design. John Wiley \& Sons, Inc. New York, NY, USA, 1995.

[2] A. R. Teel, "A nonlinear small gain theorem for the analysis of control systems with saturation," Automatic Control, IEEE Transactions on, vol. 41, no. 9, pp. 1256-1270, 1996.

[3] F. Mazenc and L. Praly, "Adding integrations, saturated controls, and stabilization for feedforward systems," IEEE Transactions on Automatic Control, vol. 41, no. 11, pp. 1559-1578, 1996.

[4] M. Jankovic, R. Sepulchre, and P. Kokotovic, "Constructive Lyapunov stabilization of nonlinear cascade systems," IEEE Transactions on Automatic Control, vol. 41, no. 12, pp. 1723-1735, 1996.

[5] L. Praly, R. Ortega, and G. Kaliora, "Stabilization of nonlinear systems via forwarding $\bmod \left\{\mathrm{L}_{i} \operatorname{sub}_{i} \mathrm{~g}_{i} / \mathrm{sub}_{i} \mathrm{~V}\right\}$," Automatic Control, IEEE Transactions on, vol. 46, no. 9, pp. 1461-1466, 2002.

[6] P. Kokotović and M. Arcak, "Constructive nonlinear control: a historical perspective," Automatica, vol. 37, no. 5, pp. 637-662, 2001.

[7] Z. Pan, K. Ezal, A. Krener, and P. Kokotovic, "Backstepping design with local optimality matching," IEEE Transactions on Automatic Control, vol. 46, no. 7, pp. 1014-1027, 2001.

[8] M. Sahnoun, V. Andrieu, and M. Nadri, "Nonlinear and locally optimal controllers design for input affine locally controllable systems,” International Journal of Control, vol. 85, no. 2, pp. 159-170, 2012.

[9] R. Sepulchre, M. Jankovic, and P. Kokotovic, “Integrator forwarding: a new recursive nonlinear robust design,” Automatica, vol. 33, no. 5, pp. 979-984, 1997.

[10] D. Carnevale and A. Astolfi, "Integrator forwarding without pdes," in Decision and Control, 2009 held jointly with the 2009 28th Chinese Control Conference. CDC/CCC 2009. Proceedings of the 48th IEEE Conference on. IEEE, 2009, pp. $33-38$.

[11] S. Benachour, V. Andrieu, L. Praly, and H. Hammouri, "Adding an integration with prescribed local behavior," in Proc. of the 50th IEEE Conference on Decision and Control, 2011.

[12] R. Sepulchre, M. Janković, and P. V. Kokotović, Constructive nonlinear control, ser. Communications and Control Engineering Series. Springer-Verlag, 1997.

[13] L. Praly, Fonctions de Lyapunov, Stabilité et Stabilisation. Ecole Nationale Supérieure des Mines de Paris, 2008.

[14] V. Andrieu and C. Prieur, "Uniting two control Lyapunov functions for affine Systems," IEEE Transactions on Automatic Control, vol. 55, no. 8, pp. 1923-1927, 2010.

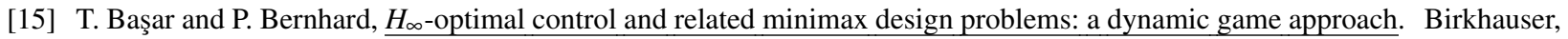
1995, vol. 5 .

[16] J. Kurzweil, "On the inversion of Lyapunov second theorem on stability of motion," Ann. Math. Soc. Trans. Ser., vol. 2, no. 24, pp. 19-77, 1956.

[17] A. Isidori, Nonlinear control systems: an introduction. Springer-Verlag New York, Inc. New York, NY, USA, 1989. 